\title{
Design of a Microstrip Diplexer and Triplexer Using Open Loop Resonators
}

\author{
A. Chinig, A. Errkik, L .El Abdellaoui, A. Tajmouati \\ LMEET Laboratory, FST of Settat Hassan $1^{\text {st }}$ University - Morocco \\ J. Zbitou, \\ LMEET Laboratory, FPK/FST of Settat Hassan $1^{\text {st }}$ University - Morocco \\ M. Latrach \\ Microwave group, ESEO, Angers France
}

\begin{abstract}
In this paper a novel design of microstrip diplexer and triplexer using open loop resonators is presented. The proposed diplexer if formed by two band-pass filters for the Industrial Scientific Medical (ISM) applications at $5.8 \mathrm{GHz}$ and the WIMAX applications (IEEE 802.16 WiMAX at 3.17 GHz to $4.2 \mathrm{GHz}$ bands). The microstrip triplexer is designed for the Digital Communication System (DCS) at $1.8 \mathrm{GHz}$, WIMAX at 3.2 GHz and for the C-band at $4.4 \mathrm{GHz}$. Each device is formed by a set of filters with a matching network by using open loop resonators and a good arrangement of the transmission feed lines. Several parametric studies were achieved to study the influence of some critical parameters on the performances of the proposed circuits. The LC equivalent circuit of the band pass filter is examined and the values of its components are calculated. ADS Agilent and CST-MWS solvers are used to predict the electrical performances of the proposed circuits. The simulated frequency responses of these structures show good results with insertion losses around $2 \mathrm{~dB}$, return losses better than $20 \mathrm{~dB}$ for all channels, and an isolation between all bands better than 20 dB. Besides, several transmission zeros are observed near the pass bands which enhance the selectivity and the isolation of the proposed devices.
\end{abstract}

Index Terms - Band pass filter, diplexer, open-loop resonators, selectivity, transmission zeros, triplexer.

\section{INTRODUCTION}

With the rapid development of the telecommunication system industry, especially the expansion of portable devices, designers are in the pursuit of new technologies and topologies to miniaturize the electronic components and to attain better electrical performances.

In order to miniaturize an electronic component, using a single antenna represents a good alternative to reduce the volume and the mass of the whole device. In this context, multiplexers and diplexers are key components in many microwave communication systems, such as radar systems, cellular phones, and satellite communication systems.

Multiplexer is a passive multiport device formed by a set of filters and a common junction. The main role of this device is to combine or to separate signals that have different frequency bands. 
While the diplexer represents the simplest form of a multiplexer. It allows a simultaneous transmission and reception of signals by using a single antenna. [1]

The development and the proliferation of standards, has led to the emergence of various innovations in diplexers/multiplexers technologies. Therefore, multi-services and multi-bands communication systems are searching new multiplexers and diplexers with high performances, low losses, high isolation, low cost and small size.

In the past, several technologies have been proposed in the literature to realize microwave components. Microwave devices particularly, filters, diplexers and multiplexers using waveguides and dielectric resonators are widely used for their high performances and the high power they can handle [2]-[3]-[4]-[5]-[6]-[7]. However, they are too heavy and bulky and their fabrication processes are too expensive.

Among all technologies used to design and to realize RF filters, microstrip technology remains the most popular due to its ease of integration with other electronic components and its compatibility with the planar fabrication process. Furthermore, this technology favors the miniaturization, since it is light, occupies a small size, and it is less expensive. Many contributions in this research area have been introduced, and several design approach have been proposed [8]-[9]-[10]-[11]-[12]. However, the main disadvantage of the microstrip technology is the low quality factor usually obtained.

In this paper, a new topology of microstrip diplexer and triplexer is proposed based on the open loop resonators. A brief study on the open loop resonator is carried to investigate the resonant frequencies and the transmission zeros of this kind of resonators by using the even and the odd-mode analysis method. Based on the open loop resonator and a good arrangement of the transmission feed lines, a band pass filter is then designed and simulated to operate at the $5.8 \mathrm{GHz}$. This microstrip structure is converted to its LC equivalent model based on a detailed method discussed in this work. After the design of this microstrip BPF another filter is designed to operate at $3.2 \mathrm{GHz}$ and combined with the first one to form the proposed microstrip diplexer. Several parametric studies were achieved to examine the influence of some critic parameters on the behavior of the proposed band pass filter and the diplexer. Finally, and according to the same method used to design the microstrip diplexer, a microstrip triplexer is then designed to operate at $1.8 \mathrm{GHz}, 3.2 \mathrm{GHz}$ and $4.4 \mathrm{GHz}$ respectively.

\section{OPEN LOOP RESONATORS}

The microstrip open loop resonator is one of the most utilized topologies to design filters diplexers mixers and antennas [13]. Known also under the name of the $\mathrm{U}$-shaped resonator, this type of structure is just a folded transmission line resonator with a gap in one of its sides as shown in Fig. 1.a.

Due to its numerous advantages including compact size and versatility, great number of microwave components using open loop resonators have been widely reported [14]-[15]-[16]-[17]. However, the main disadvantage of this kind of resonators is the presence of high order of harmonic, which limits obtaining wide stop bands. 


\section{A. Resonant Frequencies}

The main objective of this section is to study the resonance modes of the open loop resonator and its applications in the design of the microwave band pass filters.

Fig. 1.a represents the proposed open loop resonator. This conventional resonator is obtained by folding a straight-line resonator characterized by a total electrical length $\theta_{\mathrm{T}}$ and an impedance characteristic Z. Since the structure is symmetric, it can support the even and the odd modes. Based on a previous work [18], it was proved that the behavior of the open loop resonator can be deduced by analogy to that of the straight-line resonator. The resonance occurs when $\theta_{T}=n \pi$ in other terms when the length of this resonator is multiple of $\lambda / 2$ where the $\lambda$ is the wavelength and $\theta_{T}=\beta l$. In this work, in order to derive the resonant frequencies of the open loop resonator, the even and the odd-mode analysis method is used. It can be noticed also that the presence of the end-coupled arms of the open loop resonators increase the load capacitance and create tunable transmission zeros [19].

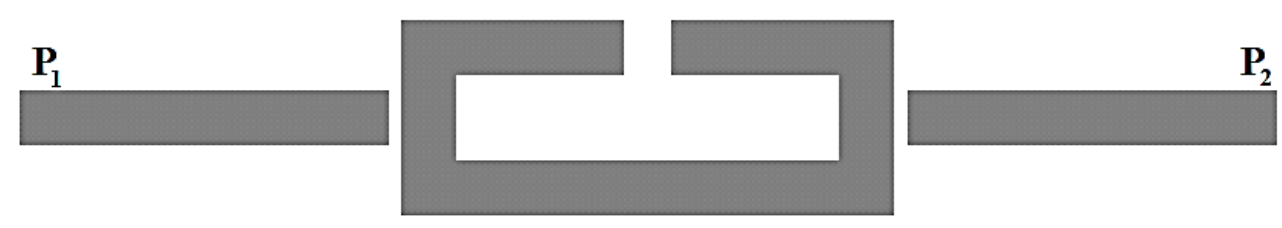

(a)

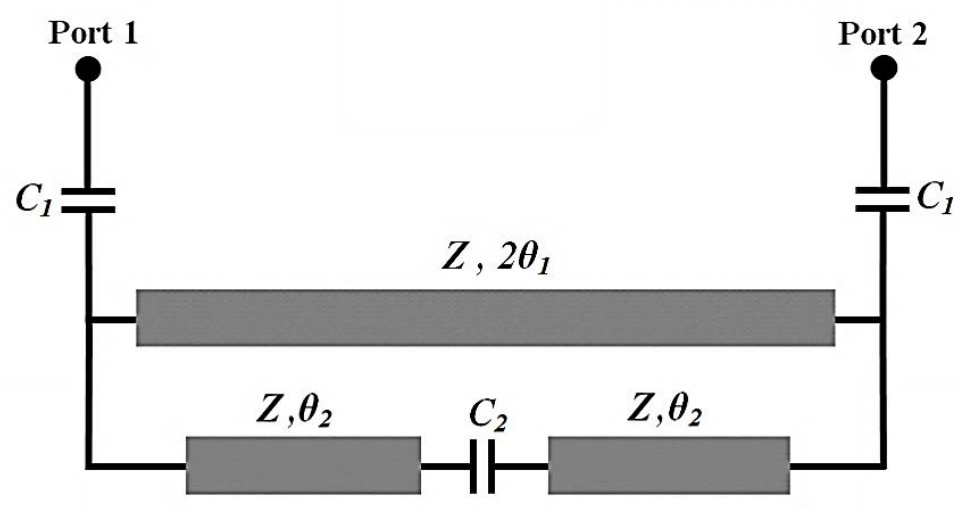

(b)

Fig. 1. (a) Schematic of the open loop resonator (b) The equivalent transmission line model.

First, the Fig. 1.b gives the equivalent transmission line model of the open loop resonator. The Fig. 2 shows the even-mode and the odd-mode equivalent transmission line model. $\mathrm{C}_{1}$ is used to model the coupling capacitance between the resonator and the input/output ports. The coupling between the two transmission lines of the resonator is modeled by the capacitance $C_{2}$. Since the line width of the proposed resonator is constant the characteristic impedance is uniform, thus the open loop resonator can be modeled by two transmission line with the same characteristic impedance $\mathrm{Z}$ and two different electrical lengths $\theta_{1}, \theta_{2}$. 


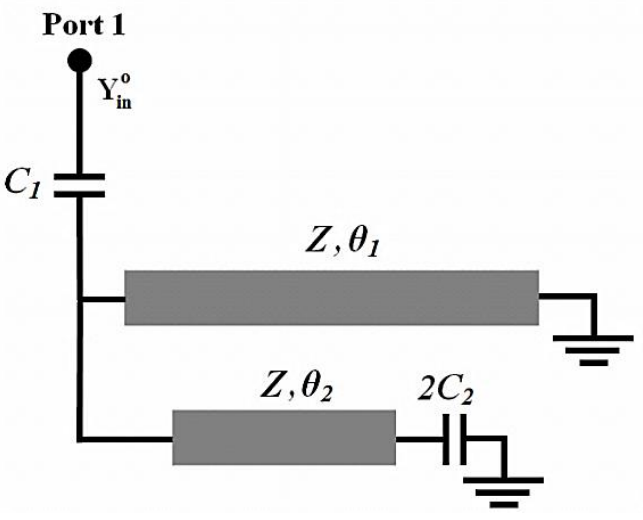

(a)

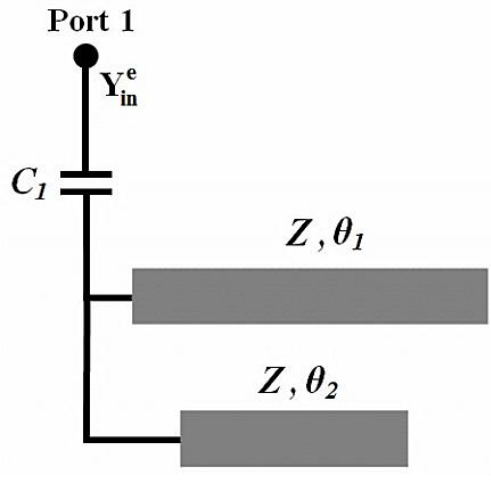

(b)

Fig. 2. (a) The odd-mode equivalent circuit (b) The even-mode equivalent circuit.

In this analysis approach, the resonant frequency $f_{0}$ denote the first resonant frequency in the oddmode while $f_{1}$ represents the first resonant frequency in the even-mode. Based on the even and the odd mode equivalent models, the odd mode input admittance can be given by:

$$
Y_{i n}^{o}=j \frac{\omega C_{1}\left[\left(2 \omega C_{2} Z+\tan \theta_{2}\right)+\cot \theta_{1}\left(2 \omega C_{2} Z \tan \theta_{2}-1\right)\right]}{2 \omega C_{2} Z+\tan \theta_{2}+\left(2 \omega C_{2} Z \tan \theta_{2}-1\right)\left(\cot \theta_{1}-\omega C_{1} Z\right)}
$$

The resonance occurs when

$$
Y_{\text {in }}^{o}=0
$$

Otherwise, for a given value of $\mathrm{C}_{2}, \mathrm{Z}, \theta_{1}$, and $\theta_{2}$ the resonant frequencies can be obtained by the following expression:

$$
\left(2 \omega C_{2} Z+\tan \theta_{2}\right)+\cot \theta_{1}\left(2 \omega C_{2} Z \tan \theta_{2}-1\right)=0
$$

In the same way, the input admittance for the even mode can be determined as follow:

$$
Y_{\text {in }}^{e}=j \frac{\omega C_{1}\left(\tan \theta_{1}+\tan \theta_{2}\right)}{\left(\tan \theta_{1}+\tan \theta_{2}+\omega C_{1} Z\right)}
$$

Similarly, the resonant condition for the proposed structure in the even mode is expressed as follow:

$$
\tan \theta_{1}+\tan \theta_{2}=0
$$

It is known that the transmission zeros appears near the pass band when the parameter $S_{21}=0$. The even and the odd mode analysis can give the expression of the $S_{21}$ based on the odd- and the evenmode input admittances. The expression of the $S_{21}$ is given by:

$$
S_{21}=\frac{Y_{i n}^{o} Y^{o}-Y_{i n}^{e} Y^{o}}{\left(Y_{i n}^{o}+Y^{o}\right)\left(Y_{i n}^{e}+Y^{o}\right)}
$$

Where $Y_{\mathrm{o}}$ represents the characteristic admittance. The condition that ensures the appearance of the transmission zeros can be deduced from the equation (6). Thus, we can write:

$$
Y_{i n}^{o} Y^{o}-Y_{i n}^{e} Y^{o}=0
$$


In other words, this condition can be expressed as fellow:

$$
Z \omega C_{2}\left(\sin 2 \theta_{1}+\sin 2 \theta_{2}\right)-\cos ^{2} \theta_{2}=0
$$

\section{B. LC model of the proposed open loop band pass filter}

The design approach of the microstrip diplexer begins generally by the design of two band pass filters. The Fig. 3 presents the proposed topology of the microstrip band-pass filter. As can be clearly seen this structure consists of a conventional rectangular open loop resonator and two Input/output feed lines. The coupling between the two transmission lines and the resonator is achieved by the gap $S_{2}$ while the gap $S_{1}$ separates the two feed lines. In this case, the folded microstrip resonator and the good arrangement of the two feed lines ensure a better reduction in size.

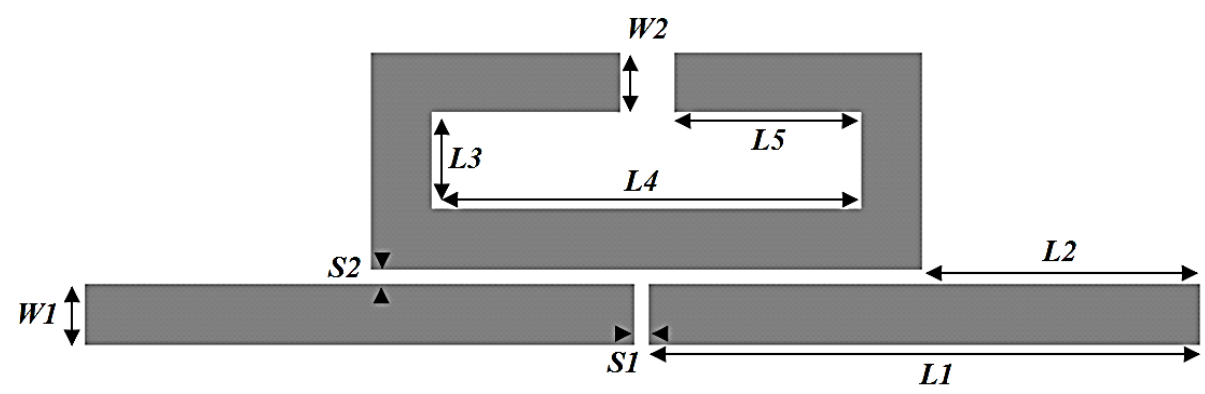

Fig. 3. The proposed band pass filter based on open loop resonator.

The dimensions of the proposed band pass filter using open loop resonator are: $\mathrm{W}_{1}=0.75, \mathrm{~W}_{2}=0.75$, $\mathrm{S}_{1}=0.2, \mathrm{~S}_{2}=0.2, \mathrm{~L}_{1}=7, \mathrm{~L}_{2}=3.6, \mathrm{~L}_{3}=1.25, \mathrm{~L}_{4}=5.5, \mathrm{~L}_{5}=2.4$, all in $(\mathrm{mm})$.

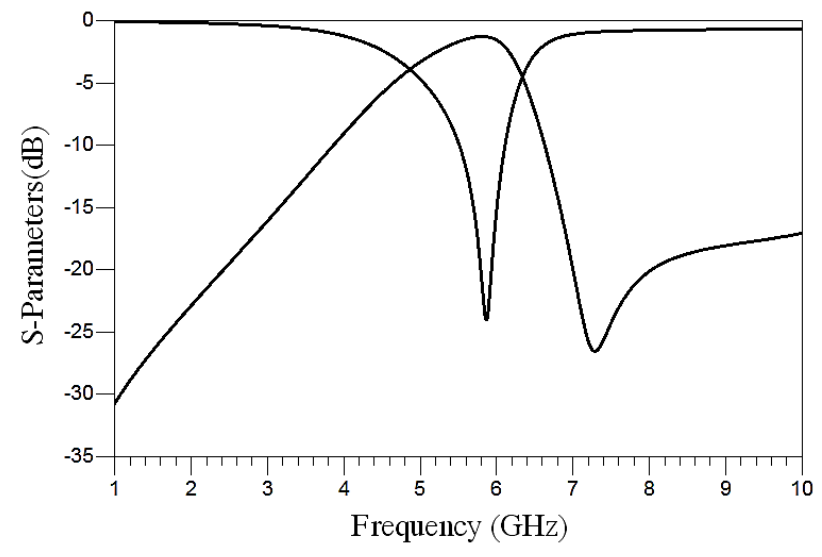

Fig. 4. Simulation results of the proposed band pass filter.

To investigate the operation of the proposed band pass filter, the designed circuit is simulated using the EM simulator ADS Agilent. From the Fig. 4 it can be seen that the band pass filter has a center frequency at $5.8 \mathrm{GHz}$, a fractional bandwidth of $19.6 \%$, a return loss of $24 \mathrm{~dB}$, and an insertion loss of $1.26 \mathrm{~dB}$. Moreover, the band pass filter presents a transmission zero near the pass band edge located at $7.28 \mathrm{GHz}$ with an attenuation of $26.6 \mathrm{~dB}$.

In order to understand the behavior of the proposed band pass filter, a second approach of this work is dedicated to study this topology by converting the microstrip structure to its equivalent LC model as shown in the Fig. 5. 
Since the proposed structure is symmetric, converting the microstrip transmission lines to the LC model is easy by using the half circuit LC analysis. The equivalent LC model is approximate and the microstrip transmission lines are considered lossless. In this LC model, the parameters $\mathrm{L}_{1}, \mathrm{~L}_{2}, \mathrm{~L}_{3}, \mathrm{~L}_{4}$, $\mathrm{L}_{5}, \mathrm{~L}_{6}, \mathrm{~L}_{7}, \mathrm{~L}_{8}$, and $\mathrm{L}_{9}$ represent the inductances of central transmission lines; $\mathrm{C}_{1}$ is used to model the capacitance effect of the gap between the two feed lines while $\mathrm{C}_{2}$ and $\mathrm{C}_{3}$ represent the capacitance effects of the gap between the feed line and the resonator. $\mathrm{C}_{8}$ and $\mathrm{C}_{9}$ represent the capacitance effects of the open stubs with respect to the ground. Furthermore, the parameters $\mathrm{C}_{3}, \mathrm{C}_{4}, \mathrm{C}_{5}$ and $\mathrm{C}_{6}$ represent the capacitance effects of the bends.

After dividing the microstrip structure to small parts in order to facilitate the conversion process, every part of the microstrip line is then converted to its equivalent LC component. The calculation method described in the following section is used to obtain the electrical values of each component. Finally, and for a better matching between the simulations results of the two circuits, some tuning techniques are employed.

To calculate the values of each component in the LC model, the equations (9) and (10) are used.

$$
\begin{gathered}
l=C Z_{c} v_{p} \\
l=\frac{L v_{p}}{Z_{c}}
\end{gathered}
$$

Parameters $l, \mathrm{C}$ and $\mathrm{L}$ represent the length, the capacitance and the inductance of a microstrip line, if the line width is considered constant. $\mathrm{Z}_{\mathrm{c}}$ is the characteristic impedance and $v_{\mathrm{p}}$ represents the phase velocity.

However, calculating the $\mathrm{C}$ and $\mathrm{L}$ values needs the calculation of $\mathrm{Z}_{\mathrm{c}}$ and $v_{\mathrm{p}}$. Once the effective dielectric constant $\varepsilon_{\mathrm{re}}$ is determined, the phase velocity is given by:

$$
v_{p}=\frac{c}{\sqrt{\varepsilon_{r e}}}
$$

$\mathrm{c}$ represents the velocity of light in free space.

The effective dielectric constant $\varepsilon_{\mathrm{re}}$ and the characteristic impedance $\mathrm{Z}_{\mathrm{c}}$ can be determined by the following equations:

For $W / h \leq 1$

$$
\begin{gathered}
\varepsilon_{r e}=\frac{\varepsilon_{r}+1}{2}+\frac{\varepsilon_{r}-1}{2}\left\{\left(1+12 \frac{h}{W}\right)^{-0.5}+0.04\left(1-\frac{W}{h}\right)^{2}\right\} \\
Z_{c}=\frac{\eta}{2 \pi \sqrt{\varepsilon_{r e}}} \ln \left[8 \frac{h}{W}+0.25 \frac{W}{h}\right]
\end{gathered}
$$

For $W / h \geq 1$

$$
\varepsilon_{r e}=\frac{\varepsilon_{r}+1}{2}+\frac{\varepsilon_{r}-1}{2}\left(1+12 \frac{h}{W}\right)^{-0.5}
$$




$$
Z_{c}=\frac{\eta}{\sqrt{\varepsilon_{r e}}}\left[\frac{h}{W}+1.393+0.677 \ln \left\{\frac{W}{h}+1.444\right\}\right]^{-1}
$$

Where $w$ is the width of the microstrip line, $h$ and $\varepsilon_{\mathrm{r}}$ represent the thickness and the permittivity of the substrate, $\varepsilon_{\mathrm{re}}$ is the effective permittivity, the parameter $\eta=120 \pi \Omega$ is the wave impedance in free space.

Each right angle bend in the microstrip open loop resonator can be modeled by an equivalent $\mathrm{T}$ network as described in [20]. In this work, an approximate form to evaluate the capacitance can be given by the expressions (16) and (17).

$$
\begin{gathered}
\frac{C}{W}(p F / m)=\frac{\left(14 \varepsilon_{r}+12.5\right) W / h-\left(1.83 \varepsilon_{r}-2.25\right)}{\sqrt{W / h}}+\frac{0.02 \varepsilon_{r}}{W / h} \text { for } W / h<1 \\
\frac{C}{W}(p F / m)=\left(9.5 \varepsilon_{r}+1.25\right) W / h+5.2 \varepsilon_{r}+7 \text { for } W / h>1
\end{gathered}
$$

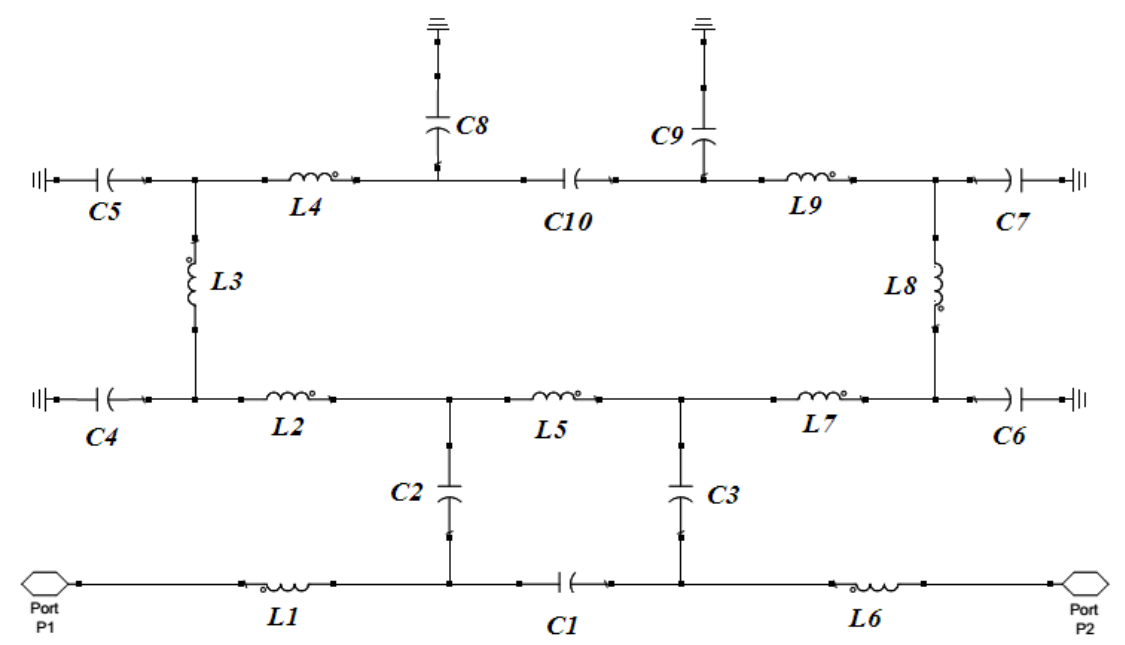

Fig. 5. The LC model of the proposed band pass filter.

The two primordial parameters that should be calculated are the effective dielectric constant and the characteristic impedance, thus for a line width equal to $0.75 \mathrm{~mm}$ we obtain $\left(\varepsilon_{\mathrm{re}}=3.05, \mathrm{Z}_{\mathrm{c}}=97.7 \Omega\right)$. After the application of the calculation method, the next step is the simulation of the converted circuit in a schematic environment. In order to get a better matching between the simulations results of the EM and the converted circuit, several simulations were achieved by tuning the obtained values of each LC model component. The EM simulator ADS was used to simulate and to compare the simulated results of the two circuits in the same frequency range. Fig. 6 demonstrates the simulated results of the LC model and the microstrip band pass filter. As seen in the figure, it can be observed that there is a good agreement between the two frequency responses. The final values of the LC components used in circuit simulation are as follow:

$$
\mathrm{L}_{1}=3.85, \mathrm{~L}_{2}=1.5, \mathrm{~L}_{3}=0.7, \mathrm{~L}_{4}=1.3, \mathrm{~L}_{5}=0.12, \mathrm{~L}_{6}=3.85, \mathrm{~L}_{7}=1.5, \mathrm{~L}_{8}=0.7 \text { and } \mathrm{L}_{9}=1.3 \text { (all in } \mathrm{nH} \text { ). }
$$
$\mathrm{C}_{1}=0.05, \mathrm{C}_{2}=0.15, \mathrm{C}_{3}=0.15, \mathrm{C}_{4}=0.032, \mathrm{C}_{5}=0.032, \mathrm{C}_{6}=0.032, \mathrm{C}_{7}=0.032, \mathrm{C}_{8}=0.14, \mathrm{C}_{9}=0.14, \mathrm{C}_{10}=0.015$ (all in $\mathrm{pF}$ ). 


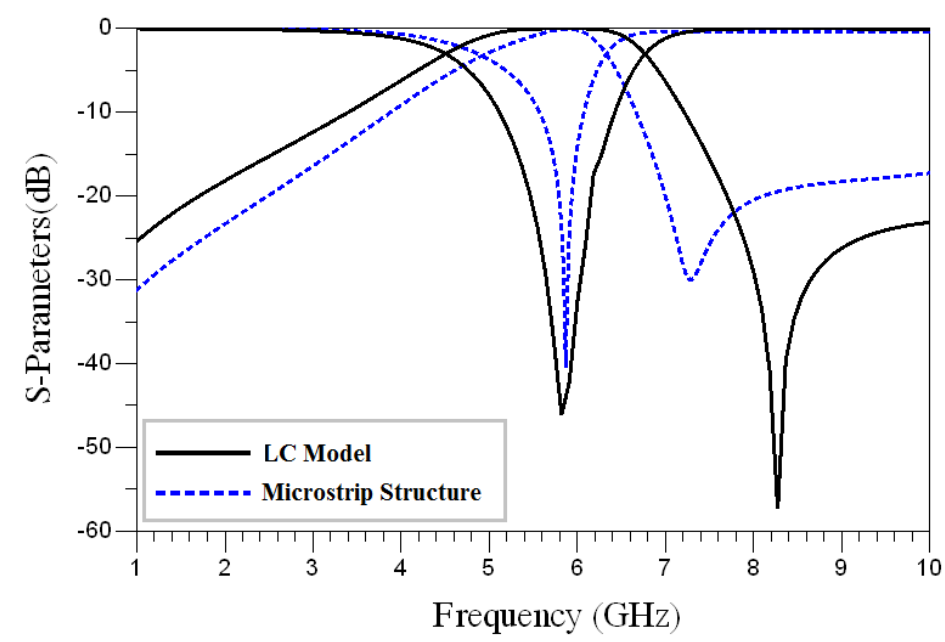

Fig. 6. Simulated results of the LC model and the Microstrip band pass filter.

One of the essential studies to understand the behavior of the proposed band pass filter is to vary the dimensions of some geometric parameters of the proposed structure, which represents an interesting way to optimize the performance of the filter.

The gap $S_{1}$ between the two feed lines was found to be a critical design parameter. In this context, various simulations were achieved to examine the frequency responses of the filter under different values of the gap $S_{1}$.

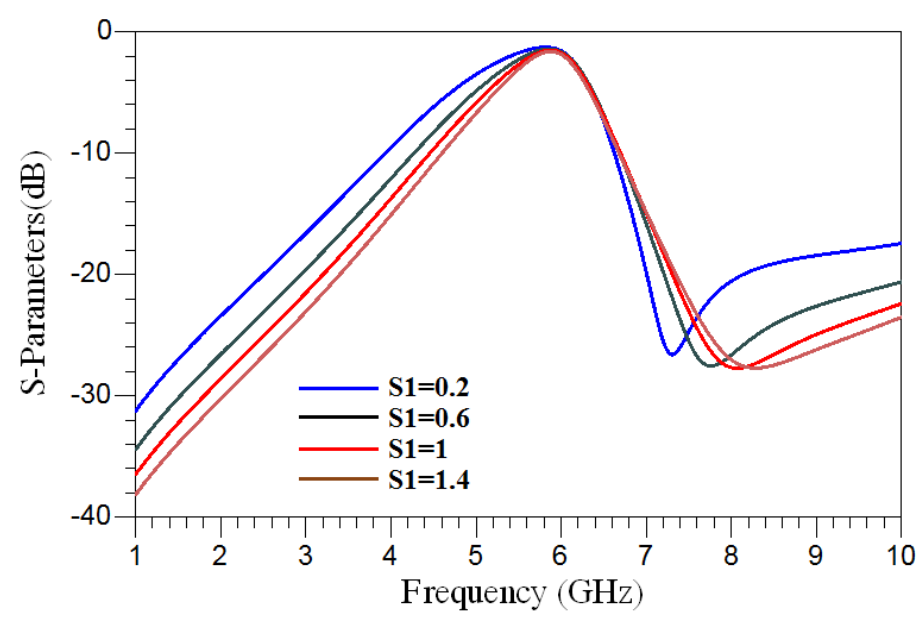

Fig. 7. The simulated $S_{21}$ parameter for different values of the gap $S_{1}$.

Fig. 7 represents the simulation results of the parameter $\mathrm{S}_{21}$ for different gap widths. From the Fig. 7 , two effects can be observed. The first is related to the bandwidth. When the gap increases, a smaller bandwidth is observed with a slight increase in the insertion losses. This is can be attributed to the reduction of the periphery between the feed lines and the resonator. In other hand, varying the gap width can affect the location and the attenuation of the transmission zero. As can be seen, when the gap width is increased the location and the attenuation of the transmission zero increase, thus signifying a better selectivity. The presence of this transmission zero is a result of the cross coupling between the two end coupled arms of the resonator as well as between the two input/output lines. Therefore, changing the gap can explains the behavior obtained. 
Varying the gap alter also the return loss of the filter. To have a clear idea on the insertion loss of the band pass filter and its selectivity, the unloaded quality factor $\mathrm{Q}_{0}$ of the band pass filter calculated from the equation (19), can envisage a measure of its electrical performances [21].

$$
\begin{gathered}
Q_{L}=\frac{1}{\frac{2}{Q_{e}}+\frac{1}{Q_{0}}}=\frac{f_{0}}{(\Delta f)_{3 d B}} \\
Q_{0}=\frac{Q_{L}}{\left(1-10^{-L / 20}\right)}
\end{gathered}
$$

with $f_{0}$ is the resonant frequency, $(\Delta \mathrm{f})_{3 \mathrm{~dB}}$ is the $-3 \mathrm{~dB}$ bandwidth of the pass band, $\mathrm{L}$ is the insertion loss in decibel and $Q_{\mathrm{L}}$ is the loaded $Q$ obtained by the equation (18). $Q_{\mathrm{e}}$ is the external quality factor.

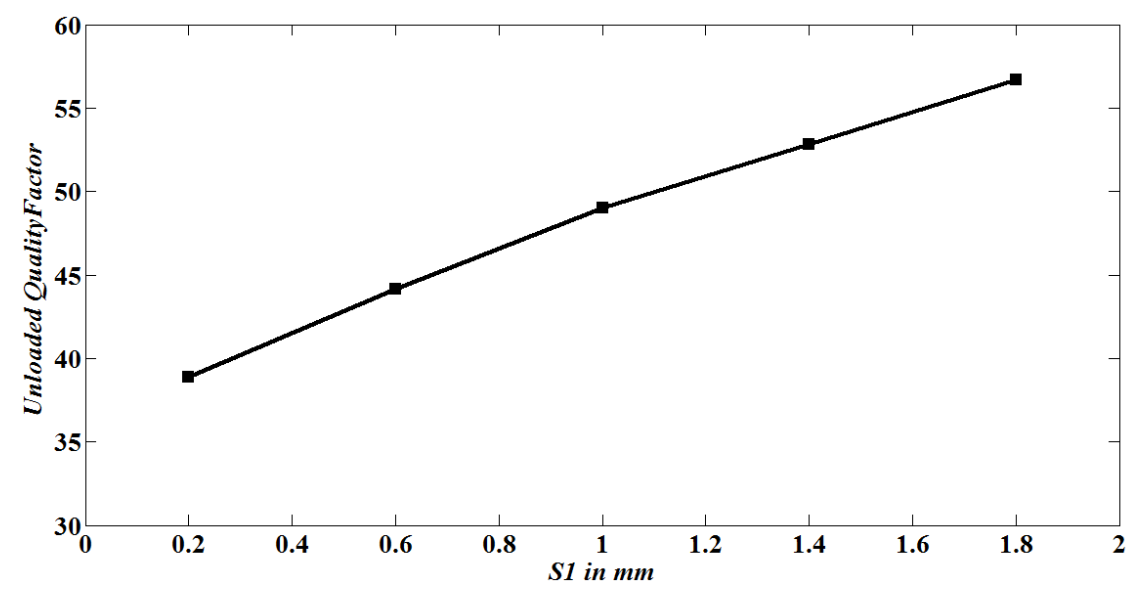

Fig. 8. The evolution of the simulated unloaded quality factor for different values of the gap $S_{1}$.

From the Fig. 8, it can be observed that the unloaded quality factor increases when the gap between the two feed lines increases. When the gap width increases from $0.2 \mathrm{~mm}$ to $1.8 \mathrm{~mm}$, the overall size of the filter increases and that generates an increase in the radiation loss and a decrease in conduction loss. So, these results can be attributed to the decrease of the current density which leads to a high unloaded quality factor [21].

\section{DESIGN OF MICROSTRIP DIPLEXER.}

Based on the open loop resonator shown in Fig. 3, and according to the position of the microstrip feed lines, it was proved that this kind of structure can be applied to design a band pass filter operating at $5.8 \mathrm{GHz}$. Similarly, another band pass filter operating at $3.2 \mathrm{GHz}$ was designed using the FR4 substrate with a thickness of $1.6 \mathrm{~mm}$, a relative electric constant of 4.4, a loss tangent of 0.025 and a conductor thickness of $35 \mathrm{um}$. The size of the proposed diplexer is extremely small, with an area equal to $(24 \mathrm{~mm} \times 22 \mathrm{~mm})$. 


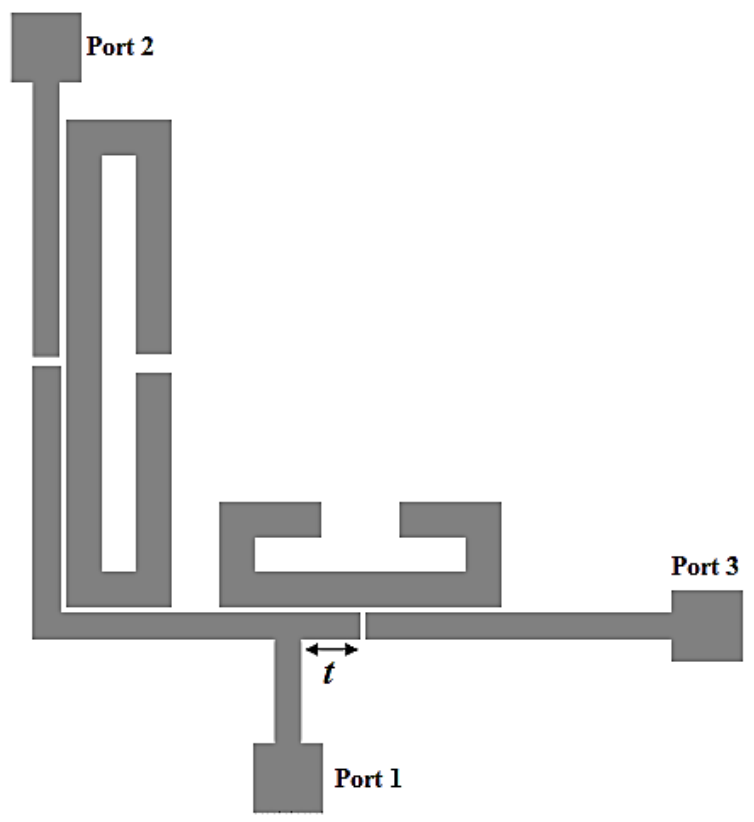

Fig. 9. Layout of the proposed microstrip diplexer.

The classical approach to design a diplexer starts with the design of two band pass filters operating at different pass bands. After that, it is essential to combine them with a matching network. This part of the circuit is so important in the design procedure, since combining the two band pass filters engenders generally a degradation in the diplexer performances. The solution used in this work involves keeping the dimensions of the junction constant during the design of each band pass filter. When, it comes to combine the two BPFs, a careful adjustment of some geometric parameters is necessary for the two resonators. The dimensions of the junction and the input/output feed lines remain unchanged.

The proposed diplexer is shown in Fig. 9. As can be seen this structure consists of a matching network based on tapped feed line connected to the port 1, two transmission lines representing the input/output ports, and two open loop resonators tuned to operate at the desired resonant frequencies. The two resonators allow the passage of the energy to the two input/output feed lines through cross coupling.

According to the analysis performed on the band pass filter in the first section, it can be concluded that this kind of resonator has specific performances. This pseudo-elliptic topology presents a transmission zeros near the pass band that will improve the rejection in the stop band and enhance the isolation of the proposed diplexer. Moreover, this structure is suitable to design a band pass filter with a minimum loss in the band pass and a wide bandwidth. Therefore, it is possible to realize a microstrip diplexer with high rejection level and good electrical performances with a limited filter order. 


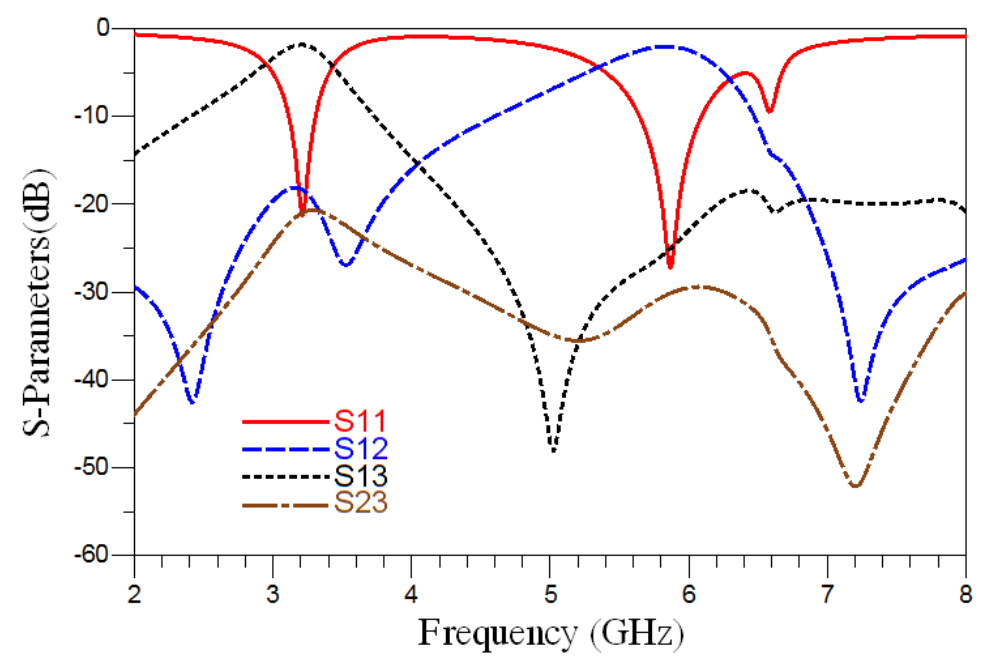

Fig. 10. Simulated results of the proposed microstrip diplexer using ADS Aglient.

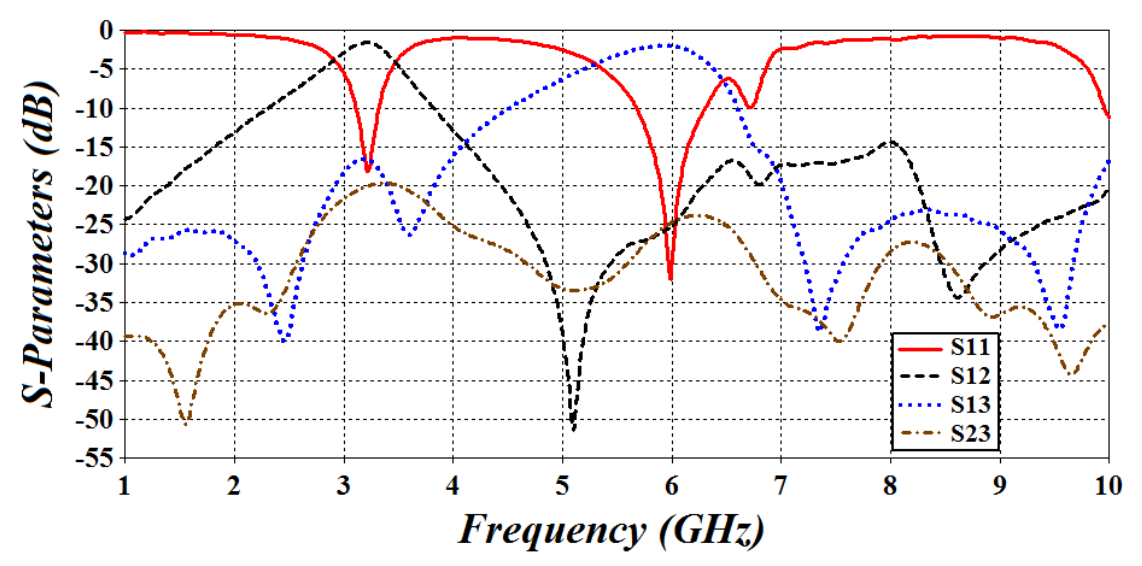

Fig. 11. Simulated results of the microstrip diplexer using CST-MWS.

The Fig. 10 represents the simulation results of the proposed diplexer. The simulated insertion loss is about $1.86 \mathrm{~dB}$ for the Rx filter and $2 \mathrm{~dB}$ for the Tx filter. The return loss in the upper and lower filter is about $27 \mathrm{~dB}$ and $21 \mathrm{~dB}$, respectively. The simulated $-3 \mathrm{~dB}$ pass bandwidth is about $8.9 \%$ and $9.75 \%$ for the high and the low band respectively. The isolation between the two channels is greater than $20.8 \mathrm{~dB}$. Moreover, several transmission zeros near the pass bands located at $2.42 \mathrm{GHz}, 3.52$ $\mathrm{GHz}, 5 \mathrm{GHz}$ and $7.25 \mathrm{GHz}$ with attenuation of $42.6 \mathrm{~dB}, 26.9 \mathrm{~dB}, 48.2 \mathrm{~dB}$ and $42.44 \mathrm{~dB}$ respectively are obtained.

It can be noticed that the EM simulator ADS Agilent is a 2D simulator. So in order to verify and to examine the electrical performances of the proposed microstrip diplexer, the CST-MWS a 3D EM simulator was utilized to design and to simulate the circuit. The simulation results obtained by this simulator are depicted in the Fig. 11. The Table I gives a resume and a comparison between the simulation results obtained by the two different simulators. Obviously, the simulated results with ADS are in good agreement with the one simulated with CST-MWS. 
TABLE I. THE SIMULATION RESULTS OF THE PROPOSED DIPLEXER UNDER DIFFERENT EM SIMULATORS.

\begin{tabular}{|l|c|c|c|c|}
\hline \multirow{2}{*}{ Parameter } & \multicolumn{2}{|c|}{ Simulation results (ADS) } & \multicolumn{2}{c|}{ Simulation results (CST) } \\
\cline { 2 - 5 } & TX & RX & TX & RX \\
\hline Frequency GHz & 3.2 & 5.84 & 3.2 & 5.9 \\
\hline Bandwidth & $312 \mathrm{MHz}$ & $515 \mathrm{MHz}$ & $405 \mathrm{MHz}$ & $657 \mathrm{MHz}$ \\
\hline Insertion loss dB & 1.86 & 2 & 1.6 & 2 \\
\hline Return loss dB & 21 & 27 & 18 & 32 \\
\hline
\end{tabular}

It is known that the external quality factor characterize the excitation of the resonators, and it depend mainly on the filter bandwidth. The external quality factor can be calculated by the equation (20).

$$
Q_{e}=\frac{2 Q_{0} Q_{L}}{Q_{0}-Q_{L}}
$$

The Fig. 12 shows the simulated external quality factor versus the position of the input feed line denoted by $t$ (Fig. 9). The position of the input feed line affects the bandwidth and the central frequency of each band pass filter.

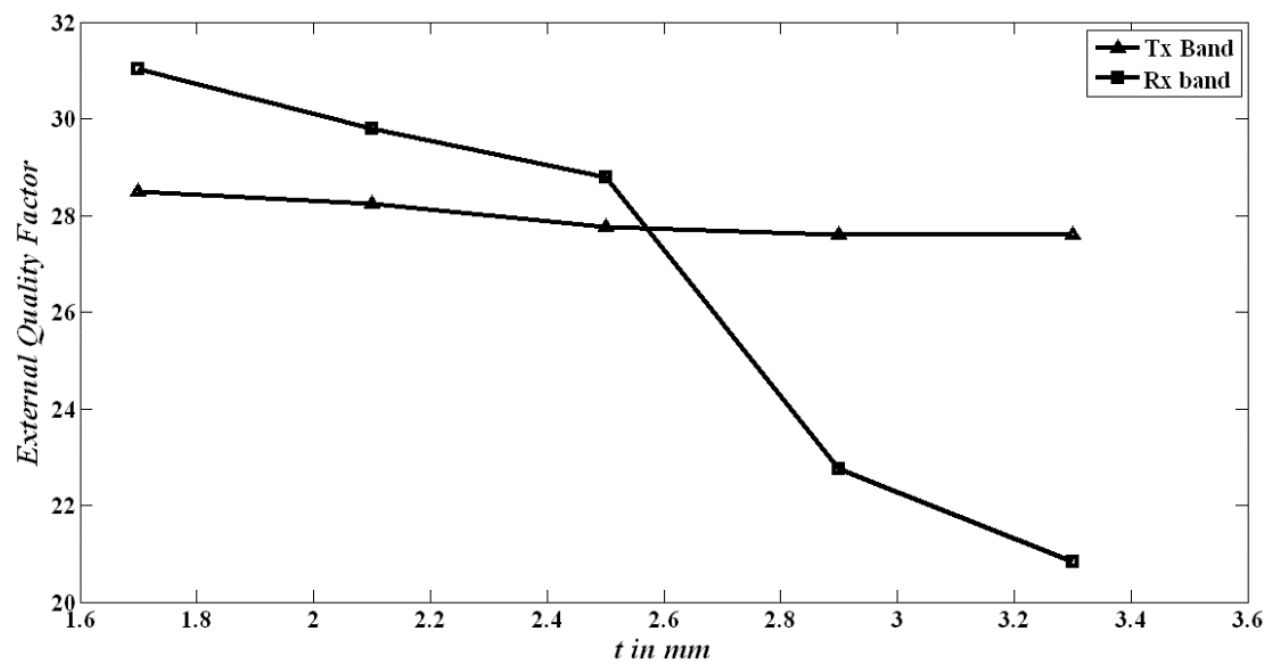

Fig. 12. The evolution of the simulated external quality factor for different values of $t$.

From the Fig. 12, it can be observed that, when $t$ is adjusted from $1.7 \mathrm{~mm}$ to $3.3 \mathrm{~mm}$; the fractional bandwidth ratio of the upper channel varies from $11.3 \%$ to $7.36 \%$ while it varies from $10.26 \%$ to $9.45 \%$ for the lower channel. The variation of the parameter $t$ has a significant impact on the fractional bandwidth of the Rx band pass filter and a slight influence on the Tx band pass filter. Thus, an important decrease is observed for the external quality factor of the Rx filter while the external quality factor of the Tx filter decreases slightly for the same variation of the parameter $t$. 
TABLE II. A COMPARISON BETWEEN THE PROPOSED DIPLEXER AND OTHER DIPLEXERS.

\begin{tabular}{|c|c|c|c|c|c|c|c|c|c|c|}
\hline & \multicolumn{2}{|c|}{$\begin{array}{c}\text { Center } \\
\text { frequency } f_{0} \text { in } \\
\text { GHz }\end{array}$} & \multicolumn{2}{|c|}{$\left|\mathbf{S}_{12}\right| /\left|\mathbf{S}_{11}\right|(\mathbf{d B})$} & \multicolumn{2}{|c|}{-3 dB FBW (\%) } & \multirow[t]{2}{*}{$\begin{array}{c}\text { Isolation } \\
\text { in }(\mathrm{dB})\end{array}$} & \multirow[t]{2}{*}{$\begin{array}{c}\text { Circuit } \\
\text { size } \\
\left(\mathbf{m m}^{2}\right)\end{array}$} & \multirow[t]{2}{*}{$\begin{array}{l}\text { Stop } \\
\text { band } \\
\text { range }\end{array}$} & \multirow{2}{*}{$\begin{array}{c}\text { Average } \\
S_{21^{-}} \\
\text {magnitude } \\
\text { of stop } \\
\text { band (dB) }\end{array}$} \\
\hline & $\begin{array}{c}\text { Tx } \\
\text { band }\end{array}$ & $\begin{array}{c}\mathbf{R x} \\
\text { band }\end{array}$ & $\begin{array}{c}\text { Tx } \\
\text { band }\end{array}$ & $\begin{array}{c}\mathbf{R x} \\
\text { band }\end{array}$ & $\begin{array}{c}\text { Tx } \\
\text { band }\end{array}$ & $\begin{array}{c}\text { Rx } \\
\text { band }\end{array}$ & & & & \\
\hline $\begin{array}{l}\text { Microstrip } \\
\text { diplexer } \\
\text { using open } \\
\text { loop } \\
\text { resonators }\end{array}$ & 3.2 & 5.84 & $1.8 / 21$ & $2 / 27$ & 9.75 & 8.9 & 21 & $24 \times 22$ & $\begin{array}{l}\text { Up to } \\
10 \\
\mathrm{GHz}\end{array}$ & 20 \\
\hline Ref[22] & 1.5 & 2 & $2.7 / 20$ & $2.8 / 20$ & 3.4 & 3.4 & 40 & $\begin{array}{c}33.9 \times 54 \\
.4\end{array}$ & $\begin{array}{l}\text { Up to } \\
8 \mathrm{GHz}\end{array}$ & 20 \\
\hline Ref [23] & 1.575 & 2.45 & $2.5 / 18$ & $1.4 / 18$ & 3 & 8 & 40 & $30 \times 70$ & $\begin{array}{l}\text { Up to } \\
6 \mathrm{GHz}\end{array}$ & 20 \\
\hline Ref [24] & 1.95 & 2.14 & $\begin{array}{c}1.22 / 1 \\
5\end{array}$ & $\begin{array}{c}1.12 / 1 \\
5\end{array}$ & 3.1 & 2.8 & 30 & $40 \times 70$ & $\begin{array}{c}\text { Up to } \\
5.6 \\
\mathrm{GHz}\end{array}$ & 30 \\
\hline
\end{tabular}

In order to have a clear idea about the electrical performances of the proposed diplexer, Table II gives a comparison between the microstrip diplexer using the open loop resonator and other microstrip diplexers proposed in literature. As it can be seen, the microstrip diplexer based on open loop resonators presents low insertion loss, wide pass band, and high return loss compared to the other diplexers. Thus, the proposed circuit presents interesting electrical characteristics. In addition, the proposed diplexer is characterized by a compact size of $24 \times 22 \mathrm{~mm}^{2}$ and a wide stop band with an attenuation of $20 \mathrm{~dB}$ up to $10 \mathrm{GHz}$, which allows it to be a very selective diplexer. However, compared to the other microstrip diplexers, the isolation between the two channel filters should be enhanced.

\section{DESIGN OF MICROSTRIP TRIPLEXER.}

The proposed microstrip triplexer is shown in the Fig. 13. This circuit is formed by connecting three band pass filters operating at different frequency bands with a matching network. Conventionally, the triplexer design need three band pass filters. Each filter can be designed individually to operate at a specific frequency band before combining it with the other channel filters. The matching network represents the main challenge in the design procedure since this part of the circuit become more complicated and can influence the frequency response of the circuit. Thus, designing a triplexer structure is more complicated than the design of the diplexer structure. Similarly, before combining the three BPFs, the dimensions of the matching network are kept constant during the design of each filter, which simplifies the design procedure of the triplexer. This method shows its efficiency since the only parameters that should be adjusted are the geometric parameters of the open loop resonators.

Based on the diplexer designed in previous section, another BPF operating at $1.8 \mathrm{GHz}$ is designed. The matching network is too large so a shift in the resonant frequencies can be observed in the two 
BPFs already designed for the proposed diplexer. The three BPFs operating at $1.8 \mathrm{GHz}, 3.1 \mathrm{GHz}$ and $4.4 \mathrm{GHz}$ are denoted channel 1 , channel 2 and channel 3 respectively.

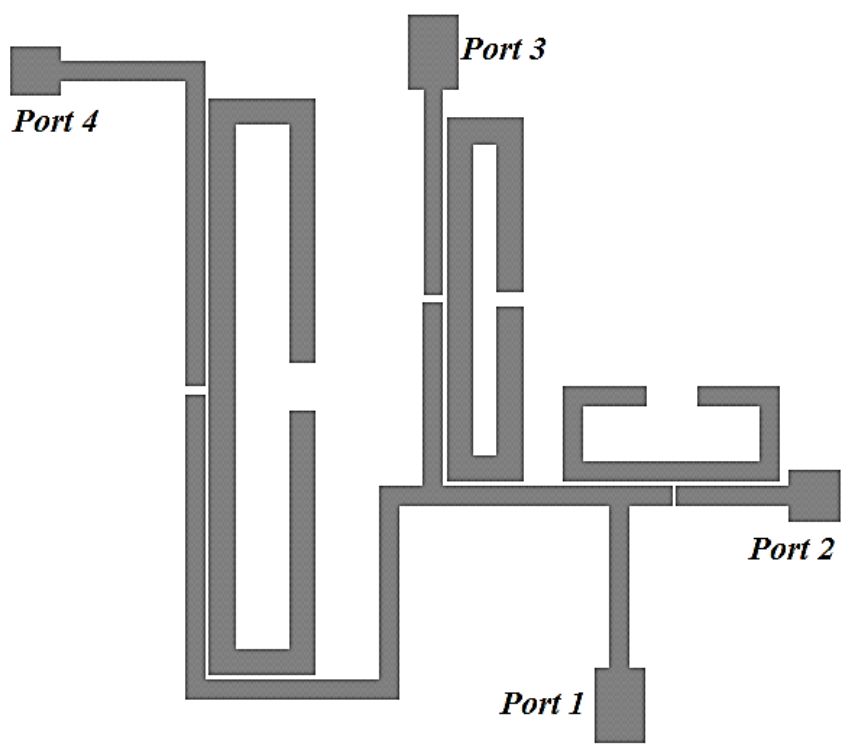

Fig. 13. The topology of the microstrip diplexer using open loop resonators.

Fig. 14 shows the simulation results of the microstrip diplexer. From the simulated results obtained by the EM simulator ADS Agilent, the insertion losses are about $1.97 \mathrm{~dB}, 1.99 \mathrm{~dB}$, and $2.3 \mathrm{~dB}$ for the cannels 1, 2 and 3 respectively. The return losses are better than $22 \mathrm{~dB}$ for the three pass bands. The fractional bandwidths of each filter are about $7.44 \%, 7.45 \%$, and $6.23 \%$ respectively. Besides, the isolation between the channels 1 and 2 is better than $21.5 \mathrm{~dB}$, while it is better than $20 \mathrm{~dB}$ between the two channels 1 and 3, concerning the isolation between the channels 2 and 3 it is about $20 \mathrm{~dB}$. Moreover, each filter presents several transmission zeros located near the pass bands. The prototype circuit size is around $33 \times 34 \mathrm{~mm}^{2}$. Table III resumes the simulated results of the proposed triplexer.

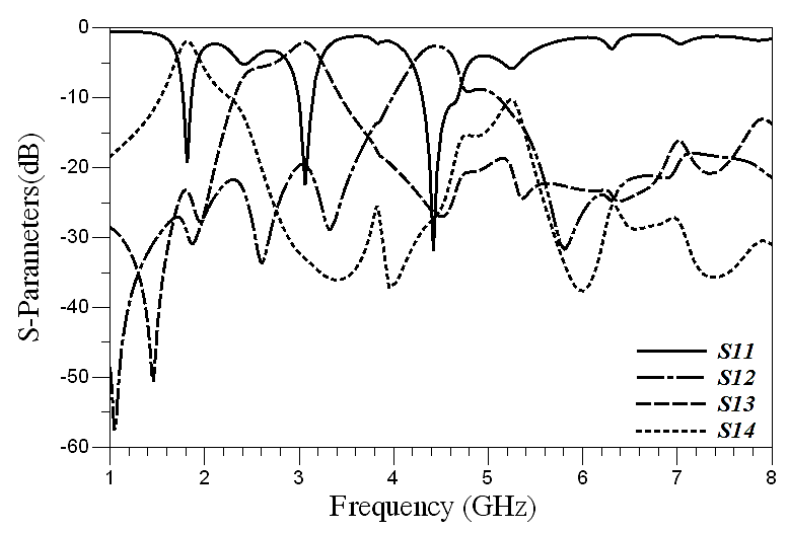

(a)

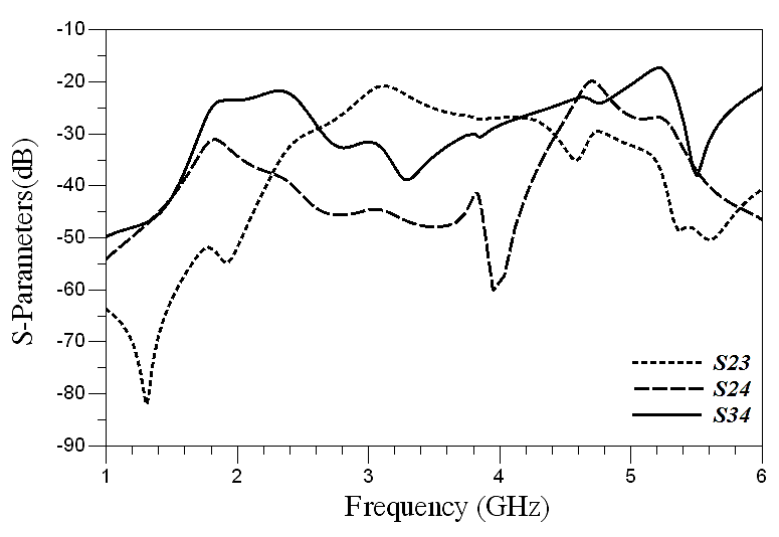

(b)

Fig. 14. Simulated results of the microstrip triplexer (a) S-parameters (b) The isolation. 
TABLE III: THE SIMULATION RESULTS OF THE MICROSTRIP TRIPLEXER USING THE ADS AGILENT.

\begin{tabular}{|l|c|c|c|}
\hline & Channel 1 & Channel 2 & Channel 3 \\
\hline Frequency GHz & 1.8 & 3.1 & 4.46 \\
\hline Bandwidth & $134 \mathrm{MHz}$ & $231 \mathrm{MHz}$ & $278 \mathrm{MHz}$ \\
\hline Insertion loss dB & 1.97 & 1.99 & 2.3 \\
\hline Return loss dB & 24 & 22 & 25 \\
\hline
\end{tabular}

\section{CONCLUSION}

In this paper, a novel compact microstrip band-pass filter structure has been proposed for designing diplexer and triplexer. It consists of an open loop resonator coupled to two Input/output feed lines. The band-pass filter with high electrical performances exhibits a quasi-elliptic function response with two transmission zeros that improve the selectivity. The derived lumped element circuit of the band pass filter can give a good estimation of the full-wave simulation response. Thus can simplify the design of circuits using open loop resonators. The parametric studies performed for the BPF and the diplexer as well as the simulated results of the proposed band pass filter, the diplexer and the triplexer have demonstrated an interesting filtering characteristics. These proposed circuits are very attractive for the ISM, DCS, WIMAX and the C-band applications. Furthermore, these circuits are very compact with an overall size of $24 \times 22 \mathrm{~mm}^{2}$ for the diplexer and $33 \times 34 \mathrm{~mm}^{2}$ for the triplexer.

\section{REFERENCES}

[1] Pozar, D. M.: Microwave Engineering. John Wiley \& Sons (1998)

[2] T. Gál, J. Ladvánszky, and F. Lénárt. "Improvement of Waveguide Diplexer Components," IEEE, Asia-Pacific Microwave Conference Proceedings. pp 28-30. 2013.

[3] Enzo Cavalieri d'Oro, Giuseppe Macchiarella “A High Power Waveguide Diplexer in Ku-band for Satellite Ground Station,” IEEE Microwave Conference, 1989. 19th European. pp 1052-1057. 1989.

[4] Skaik, T, Lancaster, M., Maolong Ke., Yi Wang, “A micromachined WR-3 band waveguide diplexer based on coupled resonator structures,” IEEE, Microwave Conference (EuMC), 2011 41st European. pp 770-773. 2011.

[5] Cheng Chen, Liu Chen, Peng Zhao. “A waveguide diplexer based on E-plane T-junction,” 15th IEEE, International Conference on Electronic Packaging Technology (ICEPT), pp 1390-1391. 2014.

[6] Bunin A. V., Vishnyakov S. V., Gevorkyan V. M., Kazantsev J. A., Mikhalin S. N., Polukarov V. I. " S, C-Band Dielectric Resonator Diplexer," IEEE, 16th Int. Crimean Conference "Microwave \& Telecommunication Technology" (CriMiCo'2006). 11-15 September, Sevastopol, Crimea, Ukraine.

[7] Bunin A.V., Vishnyakov S.V., Gevorkyan V.M., Kazantsev, J.A., Mikhalin, S.N., Polukarov, V.I. “ KU-band dielectric resonator diplexer," IEEE, 18th International Crimean Conference on Microwave \& Telecommunication Technology, CriMiCo 2008. pp 478-479.

[8] Tantiviwat S., Intarawiset N., Jeenawong R. “Wide-stopband, compact microstrip diplexer with common resonator using stepped-impedance resonators,'” IEEE, TENCON Spring Conference, pp 174-177, 2013.

[9] Xinwei Chen, Wenmei Zhang, Chunhui Yao. “Design of Microstrip Diplexer with Wide Band-stop,” IEEE, International Conference on Microwave and Millimeter Wave Technology, ICMMT '07. pp 1-3. 2007.

[10] Chi-Feng Chen, Cheng-Yu Lin, Bo-Hao Tseng, Sheng-Fa Chang. "High-Isolation and High-Rejection Microstrip Diplexer with Independently Controllable Transmission Zeros," IEEE, Microwave and Wireless Components Letters, Volume: 24. pp 851-853. 2014. 
[11] Qi Zhang, Xueguan Liu, Huiping Guo, Ying Wang. "A novel compact diplexer based on folding microstrip lines," IEEE, 4th International Symposium on Microwave, Antenna, Propagation, and EMC Technologies for Wireless Communications (MAPE), 2011. pp 785 - 788.

[12] Seungpyo Hong, Kai Chang. "'Stub-tuned Microstrip band Pass Filters for Millimeter-wave Diplexer Design,” IEEE, Microwave and Wireless Components Letters, 2005. volume 15, pp 582-584.

[13] K. Chang and L.H. Hsieh, Microwave Ring Circuits and Related Structures, $2^{\text {nd }}$ Edition. John Wiley \& Sons, New Jersey, 2004.

[14] Jia-Sheng Hong., Lancaster, M.J. "Theory and experiment of novel microstrip slow-wave open-loop resonator filters," IEEE Transactions on Microwave Theory and Techniques, 1997. vol. 45, no. 12, pp. 2358-2365.

[15] Jia-Sheng Hong, Lancaster, M.J. "Aperture-coupled microstrip open-loop resonators and their applications to the design of novel microstrip band-pass filters," IEEE Transactions on Microwave Theory and Techniques, 1999. vol. 47, no. 9, pp. 1848-1855.

[16] Cheng-Cheh Yu, Kai Chang. "Novel compact elliptic-function narrow-band band pass filters using microstrip openloop resonators with coupled and crossing lines,' IEEE Transactions on Microwave Theory and Techniques, 1998. vol. 46, no. 7, pp. 952-958.

[17] Konpang, J., Thongnoi, L. “A Compact Four-pole Cross-coupled Square Open Loop Resonator Diplexer,” IEEE, German Microwave Conference (GeMIC), 2011. pp 1-4.

[18] A.Chinig, J. Zbitou, A. Errkik, L. Elabdellaoui, A. Tajmouati, A. Tribak, M.Latrach. “A New Microstrip Diplexer Using Open-Loop Resonators,' Journal of Microwaves, Optoelectronics and Electromagnetic Applications, vol. 13, No. 2, pp 75-86. December 2014.

[19] K. S. Chin, D.J. Chen. "Novel Microstrip Bandpass Filters Using Direct-Coupled Triangular Stepped Impedance Resonators for Spurious Suppression,’’ Progress In Electromagnetics Research Letters, 2009. vol. 12, pp 11-20.

[20] J-S. Hong, Microstrip Filters for RF/Microwave Application, $2^{\text {nd }}$ ed. (Wiley, New York, 2011).

[21] Jarry, P., Beneat, J. Design and Realizations of Miniaturized Fractal RF and Microwave filter, A John Wiley \& Sons, Inc., Publication. (2009).

[22] Chen, C. F., Huang, T. Y., Chou, C. P., \& Wu, R. B. "Microstrip diplexers design with common resonator sections for compact size, but high isolation," Transactions on Microwave Theory and Techniques, IEEE, 2006. pp 1945-1952.

[23] Huang, C. Y., Weng, M. H., Ye, C. S., \& Xu, Y. X. “'A high band isolation and wide stopband diplexer using dualmode stepped-impedance resonators,” Progress In Electromagnetics Research, 2010. 100, pp 299-308.

[24] Chuang, M. L., \& Wu, M. T. “Microstrip diplexer design using common T-shaped resonator," Microwave and Wireless Components Letters, IEEE, 2011. pp 583-585. 